\title{
REFLEXIONES SOBRE EL CONCEPTO DE REVOLUCIÓN EN EL LIBERALISMO CONTEMPORÁNEO
}

Eric Herrán*

Por lo menos desde finales del siglo más iluminado de todos, es muy difícil meditar seriamente acerca del concepto de revolución sin reflexionar sobre la naturaleza y los alcances de la prudencia. De lo anterior se desprende que los excesos normalmente asociados con las revoluciones modernas han validado históricamente en nosotros la costumbre de oponer a estos excesos la templanza que reside en la frónesis. En vista de que podría parecer petulante afirmar que la deducción precedente es casi una banalidad, me abstendré de hacer semejante afirmación. Menos obvia y más callada, sin embargo, es esta otra conjetura, asimismo contenida en mi primer apunte: el esclarecimiento moderno de la prudencia sólo es posible por el cabal entendimiento de la revoluciones modernas.

Lo anterior no implica que las revoluciones (modernas o no) sean fáciles de interpretar ni que éstas deban constituir el foco gravitacional de la teoría política. Sobre este último punto en particular, y limitándonos al presente siglo, es claro que el estudio de las revoluciones no basta para comprender con plenitud -entre otros fenómenos-el surgimiento y la evolución de las principales rivalidades ideológicas y geo-

* Instituto Tecnológico Autónomo de México. 
ERIC HERRÁN

políticas de los últimos cuarenta años. Pero, al mismo tiempo, no puede haber un entendimiento coherente de dichos fenómenos sin un examen adecuado del carácter de los movimientos revolucionarios.

Aun suponiendo que lo dicho hasta ahora indica una argumentación plausible o por lo menos no descabellada, hay lugar para las siguientes preguntas. ¿Para qué sirve actualmente una reflexión sobre los vínculos entre revolución y prudencia, por un lado, y entre revolución e interpretación política, por el otro, cuando parece que la "época" de las grandes revoluciones se ha extinguido con rapidez a partir de 1989 ? Es decir, ¿para qué sirve semejante reflexión ahora que la revolución ha dejado de ser un ideal creíble (ya no digamos deseable)? $\mathrm{O}$, en palabras que van todavía más al punto, ¿qué relevancia puede tener hoy en día una investigación sobre el concepto de revolución para la tarea de escudriñar el futuro político de la democracia liberal?

Sin duda, cuestiones de este tipo ocupan actualmente -y con razóna buena parte del pensamiento político de izquierda, en el afán de este último por elucidar la naturaleza de una teoría crítica dentro de los límites de la razón democrática. No puede menospreciarse el valor que tanto para la historia de las ideas como para la teoría política del

166 siglo veinte encierran tales revisiones. Sin embargo, no es en la perspectiva de la izquierda que a menudo han surgido en tiempos recientes las propuestas más interesantes en torno a la existencia de un vínculo esencial entre algún tipo de pensamiento revolucionario y las aspiraciones de la democracia liberal. Si la rehabilitación de una cierta idea normativa de la revolución es aún posible y deseable en el horizonte actual de la democracia liberal, quizá los esfuerzos más prometedores en este sentido no provienen del fondo de las doctrinas políticas que han estado de suyo ligadas con la idea de revolución, sino de aquéllas que tradicionalmente -al menos en este siglo- se han pensado a sí mismas como incompatibles con el discurso revolucionario. ${ }^{2}$ Este plan-

${ }^{1}$ Cfr. John Dunn, Interpreting Political Responsibility, 1990, Cambridge, Polity Press, p. 94.

${ }^{2}$ Aunque de signo contrario, se percibe aquí una situación análoga a la del relato ofrecido por François Furet a propósito de lo acontecido en los años 
teamiento alude principalmente, en su parte última, al liberalismo político. Alude, en concreto, al liberalismo revolucionario defendido por Bruce Ackerman, profesor emérito de derecho y de ciencia política en la Universidad de Yale.

Los primeros acontecimientos sociales y políticos que tienen lugar en Europa oriental inmediatamente después del otoño de $1989^{3}$ autorizan, según Ackerman, la invocación de una circunstancia observada hace casi un siglo y medio: un "fantasma", el de la revolución, recorre el viejo continente. Pero a diferencia de la observación hecha en el más célebre de todos los manifiestos, la de Ackerman puntualiza las siguientes dos cosas. La primera es que las andanzas del fantasma no se circunscriben a los escenarios europeos; la segunda, que el espectro revolucionario en cuestión no es el del comunismo, sino el de la democracia liberal. El re-surgimiento del liberalismo revolucionario, añade, no ha dejado de sorprender incluso a los propios liberales. La razón del asombro de estos últimos radica, a fin de cuentas, en la identificación espuria del liberalismo político contemporáneo con una aspiración antirrevolucionaria. Aunque ilegítima, afirma Ackerman, esta identificación no deja de ser históricamente explicable.

setenta en el seno de la intelectualidad francesa, en ese entonces mayoritariamente de izquierda. En Pensar la Revolución Francesa-texto de 1977Furet observa el enorme descrédito en el cual se hundían ya los ideales (y los ídolos) revolucionarios. Lo interesante al respecto, señalaba Furet, es que la crítica de la revolución había dejado de constituir el monopolio de la derecha y se convertía en un tema central de la reflexión de gauche. Era el repudio del ideal revolucionario por parte de los intelectuales izquierdistas, lo que en aquel momento parecía ofrecer las perspectivas críticas más interesantes, por lo menos acerca del futuro de la propia izquierda.

${ }^{3}$ Año en el que, para algunos observadores, con la caída del Muro de Berlín comienza anticipadamente el siglo veintiuno. Véase, por ejemplo, Michael Ignatieff, Blood and Belonging: Journeys into the New Nationalism, 1995, Nueva York, Noonday Press, p. 4.

${ }^{4}$ Bruce Ackerman, The Future of Liberal Revolution, 1992, New Haven y Londres, Yale University Press, p. 1. 
ERIC HERRÁN

Buena parte de la culpa histórica recae en el marxismo-leninismo y su exitosa apropiación, a partir de 1917, de la idea de revolución. Apoyados en diversas circunstancias favorables, Lenin y su descendencia política fueron lo suficientemente hábiles -observa Ackerman en su peculiar estilo- para "hacer creer a casi todos que su forma de revolución era la mercancía auténtica y que las otras eran imitaciones, cuando no algo peor". 5 Si a esto añadimos que los liberales del presente siglo han salido - no sin razón-severamente "traumatizados" de su enfrentamiento no sólo con el comunismo soviético sino también con el nazismo, no es difícil comprender que los primeros hayan adoptado una inquebrantable posición antirrevolucionaria. No obstante, en el presente es preciso cuestionar la autocomplacencia que acompaña a la reproducción de este lugar común liberal que, en el nivel más general, termina asociando la idea de revolución con alguna forma de tiranía. $¿ O$ acaso debemos dejar las cosas tal como están y "permitir que unos cuantos extremistas hagan de la revolución un concepto imposible"? ${ }^{6}$

En este punto, la reflexión ackermaniana sugiere que la tarea de emancipar a la revolución del calabozo al cual ha sido reducida por su vinculación con las experiencias históricas del comunismo y el nazismo, implica una doble reconciliación. Por un lado, es necesario conjugar el pensamiento político liberal con la idea de revolución. Simultáneamente, es preciso armonizar el ideal revolucionario con el llamado estado de derecho (o el ideal de la coexistencia pacífica bajo el primado de la ley, expresado en garantías constitucionales). Sobre este último aspecto, no se piense que la cuestión punzante se centra únicamente en cómo hacer para que la fogocidad revolucionaria encuentre sus límites en el imperio de la ley. Si esto fuera todo lo que Ackerman tiene que decir al respecto, su propuesta a duras penas podría parecer original. Con respecto a la naturaleza de los nexos entre la revolución y el estado de derecho, una faceta no menos decisiva que la anterior es ésta: ¿cómo hacer para que la aspiración liberal constitucionalista "au-

\footnotetext{
${ }^{5}$ Ibid.

${ }^{6}$ Ibid., p. 6.
} 
torice" a los revolucionarios liberales a "aprovechar sus oportunidades" de transformación social? ${ }^{7}$ Es claro que el estado de derecho no sólo debe encauzar el ímpetu revolucionario; también debe ser capaz de suscitarlo.

Pero si, de un lado, liberalismo y revolución son esencialmente compatibles, y si del otro, debe ser posible armonizar la revolución con el estado de derecho, ello implica la reformulación de cada uno de los tres términos involucrados. Sobre el primero, tan sólo puedo indicar aquí la originalidad que encierra la propuesta de Ackerman en el sentido de que el reconocimiento auténtico de los pluralismos modernos obliga a pensar el liberalismo político como "una forma de hablar acerca del poder", antes que como una doctrina que establece compromisos axiomáticos de "primer orden" tales como la libertad o la equidad. ${ }^{8}$ En cuanto al tercer término -el estado de derecho-Ackerman tiene algunas propuestas interesantes que hacer, sobre las cuales trataré un poco más adelante. Puedo adelantar al respecto, no obstante, que su reconsideración de la idea del estado de derecho pone énfasis en que hasta ahora se ha subestimado "el papel creativo del constitucionalismo" y que los liberales deberían poder estar en posición de "explotar plenamente" el potencial de éste último. ${ }^{9}$ He dejado para el final el término intermedio -la revolución-que es el elemento común a las dos reconciliaciones arriba mencionadas. Ninguna de estas dos es posible sin un replanteamiento profundo del ideal revolucionario en el mundo moderno. Es por ello que, previo a la exploración de sus posibilidades democrático-liberales, se requiere de un análisis conceptual de la revolución.

\section{${ }^{7}$ Ibid., p. 3.}

${ }^{8}$ Bruce Ackerman, La Justicia Social en el Estado Liberal, trad. C. Rosenkrantz, 1993, Madrid, Centro de Estudios Constitucionales, p. 39; el énfasis es del propio Ackerman. Para un análisis de su teoría liberal véase Eric Herrán, "Bruce Ackerman o la Naturaleza y los Límites del Liberalismo Político", ponencia presentada en el Congreso Nacional de Ciencia Política, México, D.F., 25-28 de septiembre, 1996 (mimeog.).

${ }^{9}$ Bruce Ackerman, The Future of Liberal Revolution, op. cit., p. 3. 
ERIC HERRÁN

Ackerman da muestras de poseer un conocimiento suficiente de diversos estudios sobre fenómenos revolucionarios del presente y del pasado, y en consecuencia sabe que el primer deber del analista consiste en reconocer la confusión que resulta de los distintos propósitos para los cuales se echa mano del concepto de revolución. ${ }^{10}$ Quizá esta confusión es del todo inevitable, si consideramos -en palabras de John Dunn- que a diferencia de conceptos tales como "democracia o justicia o igualdad o libertad, la revolución no es en primera instancia un criterio normativo que los seres humanos oponen a la realidad social y política, y al cual pretenden que esta última se ajuste". Más bien, continúa diciendo Dunn, "la revolución es en primera instancia una característica del mundo histórico real en un tiempo y un lugar particulares". " La referencia histórica que ineludiblemente habita el concepto de revolución explica por qué éste no puede ser un concepto normativo como los otros. Pero, sin duda, para bien o para mal, es también un concepto normativo. La revolución seguramente no puede $-\mathrm{y}$ en esto Dunn quizá tiene razón-indicar una normatividad "en primera instancia". Lo cual sólo vuelve más complejo el análisis del concepto de revolución, pues si su referencia histórica "contamina" su dimensión normativa, la reformulación de la propia referencia histórica es siempre posible a la luz de puntos de vista teóricos que no pueden aislarse completamente de intereses normativos. Tal parece entonces que el análisis conceptual del fenómeno revolucionario es sólo el epítome de una situación -a saber, el problemático vínculo entre hechos (históricos o de otra clase) e interpretación (normativa o no)- que en algún punto no es posible dejar de percibir en relación al análisis de otros conceptos como los anteriormente señalados. ${ }^{12}$ De cualquier manera, lo que me interesa resaltar en esta digresión es el hecho de que Ackerman da visos de tener conciencia suficiente de estos problemas

${ }^{10}$ Ibid., p. 5.

11 John Dunn, op. cit., p. 85.

${ }^{12}$ Por lo demás, la existencia de paradigmas o eventos ejemplares históricos que constriñen fuertemente el campo de lo normativo, es quizá sólo más fácil de admitir en el caso de la revolución que en el de otros conceptos. 
y, por lo tanto, tendría que estar de acuerdo en que su elección de una cierta idea de revolución se justifica plenamente desde un punto de vista particular, que no es otro que el punto de vista del liberalismo político. Más que indicar con esto una debilidad en el esquema liberalrevolucionario de Ackerman, mi intención es la de reconocer en la adopción de dicho punto de vista su momento más sólido.

Ackerman propone una idea de revolución que, asegura, subyace a todas las ocurrencias históricas del fenómeno revolucionario. "Una revolución" - dice - "es una tentativa exitosa de transformación de los principios rectores y de las prácticas de un aspecto básico de la vida por medio de una movilización colectiva consciente." ${ }^{13}$ Todas las revoluciones, piensa Ackerman, comparten un sustrato que esta definición intenta expresar. ${ }^{14}$ Dicho sustrato sugiere, a su vez, que la acción revolucionaria conlleva una peculiar referencia al tiempo. Antes que ninguna otra cosa, los revolucionarios proponen siempre "escindir el tiempo en dos partes: un Antes y un Ahora". En esta perspectiva, es siempre posible detectar en el Antes algo que no está bien con respecto a la manera en que la gente piensa y actúa. Por lo demás, es siempre

${ }^{13}$ Ackerman, The Future of Liberal Revolution, p. 5-6. Esta idea de revolución es tan general que, a decir de Ackerman, abarca a las revoluciones científicas; ibid., p. 5. Por supuesto, tanto aquí como en momentos en que vincula la idea de revolución con la sustitución de paradigmas, Ackerman alude al tipo de filosofía de la ciencia defendida por Thomas Kuhn. Al respecto, véase Thomas S. Kuhn, La estructura de las revoluciones cientificas, trad. A Contin, 1971, México, FCE.

${ }^{14}$ Es claro, en consecuencia, que su propuesta de una revolución liberal no depende del establecimiento de una diferencia entre revoluciones premodernas y modernas. Con todo, Ackerman reconoce la relevancia que esta distinción puede tener en ciertos contextos, hasta el punto de discurrir acerca del tipo de revolución moderna que se halla implicado en su visión liberal. Al respecto, véase The Future of Liberal Revolution, p. 7-8. Sobre el fenómeno revolucionario moderno en particular, véase John Dunn, Modern Revolutions: An Introduction to the Analysis of a Political Phenomenon, 1972, Cambridge, Cambridge University Press. 
ERIC HERRÁN

Ahora, en esta lógica, que podemos escudriñar la posibilidad de corregir aquello que no está bien y poner en marcha un "nuevo comienzo". A su vez, este "nuevo comienzo" sólo puede llevarse a cabo a través de la movilización de hombres y mujeres conscientes de su participación en una acción colectiva de gran alcance. Es así que estos hombres y mujeres "reconocen la validez de nuevas verdades y prácticas - de paradigmas, si se quiere- y proceden a reorganizar su vida colectiva confiriéndole un nuevo peso a su importancia". ${ }^{15}$

$\mathrm{Al}$ momento de señalar lo anterior, Ackerman desea que no pasemos por alto que, tal como él la entiende, la acción revolucionaria no exige un cambio total en los principios rectores. Semejante cambio (más allá de los "excesos retóricos", nos dice) jamás ha sido posible y nunca lo será. Ello es especialmente cierto si adoptamos el punto de vista del liberalismo político, para el cual la delimitación de las ambiciones orientadas al cambio social posee un carácter fundamental. Un liberal, pues, no es un "totalista" (totalist), y su idea de la revolución tendrá que ser consecuente con esta premisa. ${ }^{16}$

No obstante, el repudio de la visión totalista no impide que la revolución liberal consiga eventualmente aquello que le es posible conseguir: la realización de "grandes cambios en el sistema político sin tener que transformar todos los sectores de la sociedad". Desde esta óptica, se vuelve posible reconocer como eventos revolucionarios propios de la sociedad liberal la transición de una economía de laissez-faire a una de estado benefactor, así como la transformación profunda de nuestra actitud hacia el medio ambiente (aun cuando dicha transformación deje relativamente intactas otras prácticas). Siempre y cuando, debemos

${ }^{15}$ Ackerman, The Future of Liberal Revolution, p. 5. Apenas es necesario hacer notar que en esta discusión Ackerman tiene en mente algo así como un tipo ideal del fenómeno revolucionario. Sabe, en consecuencia, lo que todos sabemos: que en el mundo real la manipulación y la inconciencia constituyen dimensiones siempre probables de la movilización colectiva. Sobre su adopción explícita de un enfoque basado en tipos ideales, véase $i b i d .$, p. 7-8. ${ }^{16}$ Ibid., p. 6. 
añadir, estos cambios ocurran por la vía de una movilización social consciente y responsable de sus actos. ${ }^{17}$

En este preciso estadio de la argumentación del profesor Ackerman, una pregunta surge con la fuerza que necesariamente le es propia. ¿Qué tanta violencia revolucionaria está dispuesta a condonar esta concepción del liberalismo político? Interpreto la respuesta de Ackerman a esta cuestión de la manera siguiente: en la normatividad (que no es todo, pero es mucho), ninguna. La revolución liberal debe poder entenderse como una movilización democrática pacífica. Para ello es urgente separar revolución de violencia. ${ }^{18}$ Cuando se ha ejercido, la violencia revolucionaria ha tenido como propósito la supresión o el debilitamiento de quienes en su pensamiento y sus acciones son percibidos como obstáculo para el programa revolucionario (cualquiera que éste sea). Ha tendido, en consecuencia, a la eliminación o el agotamiento de quienes aparecen como irreductiblemente distintos, como incurablemente otros. En su actuar, la violencia revolucionaria se opone entre otras cosas a la posibilidad del pluralismo moral y con ello contradice una de las intuiciones más elementales que subyacen a las democracias modernas. ${ }^{19}$ Por ello es necesario, repitámoslo, separar revolución y violencia. ¿O acaso, insistiría Ackerman, vamos a permi-

${ }^{17} \mathrm{Ibid}$.

${ }^{18}$ Ibid., p. 11-4; para la apreciación explícita de la revolución liberaldemocrática como una revolución pacífica, véase ibid., p. 17.

${ }^{19}$ Desde luego, por desgracia la supresión del Otro no es exclusiva de la violencia revolucionaria. Quienes desean mantener a toda costa ciertos privilegios que les otorga la perpetuación del status quo y se oponen por tanto a un cambio social significativo (con todo y que las propuestas de cambio surjan efectivamente con base en una movilización social responsable), no son menos proclives al empleo de medios violentos. Ambas formas de recurso a la violencia contradicen el espíritu pluralista e igualitario que anima a las democracias liberales.

Para Ackerman, en todo caso, la única situación que podría justificar-"tal vez"- el recurso liberal a la violencia es aquélla en la cual "la élite en el poder responde a las demandas liberales de justicia social con la supresión brutal del movimiento revolucionario"; ibid., p. 14. 
ERIC HERRÁN

tir que unos cuantos violentos "extremistas" se apropien de la idea de cambio social?

Disociar la revolución de la violencia y, en un mismo soplo, vincular la revolución con la tradición del liberalismo político, implica-como ya se ha anunciado- la reconsideración de las potencialidades dinámicas del constitucionalismo. Si la idea de revolución debe ser compatible con una constitución democrático-liberal, entonces una manera de incorporar el deseo "vanguardista" de promover "grandes cambios" sociales y de limitar al mismo tiempo las ambiciones que este deseo conlleva, consiste en "diseñar un sistema constitucional que someta a los aspirantes a revolucionarios a una serie de pruebas democráticas imparciales". ${ }^{20}$ No es posible examinar aquí en toda su complejidad la propuesta específica de diseño constitucional que Ackerman ofrece en este sentido $^{21}$-y al decir esto tengo en mente tan sólo aquella comple-

\section{${ }^{20}$ Ibid.}

${ }^{21}$ En We the People, v. 1., Foundations, 1993, Cambridge, Mass. y Londres, Harvard University Press-Belknap Press, Ackerman ofrece la esencia, el trasfondo y algunos detalles de su visión constitucionalista. Ello ocurre a propósito de una reinterpretación fundamental de la historia constitucional estadunidense. No hay duda entonces de que el potencial de universalización que abrigan sus reflexiones constitucionalistas en torno al vínculo primordial entre el liberalismo político y la aspiración revolucionaria, se encuentra de inicio restringido por su referencia particular a los Estados Unidos de América. No es menos cierto, empero, que dichas reflexiones indican la posibilidad (y hasta la exigencia) de trascender esta referencia particular en algún grado significativo. Esta indicación es evidente en el deseo de Ackerman de explorar las implicaciones, tanto teóricas como prácticas, que encierra la adopción de su enfoque liberal en el contexto de los diversos movimientos de agitación "democrática" observados en Europa oriental a partir de 1989. The Future of Liberal Revolution cristaliza este deseo.

Para una evaluación, desde diversos puntos de vista, de la reformulación del constitucionalismo estadunidense emprendida por Ackerman, véanse los artículos dedicados a su análisis que aparecen en Ethics 104, n ${ }^{\circ} 3$, abril de 1994, seguidos allí mismo por una respuesta del propio Ackerman a sus críticos. 
jidad que puede ser relevante para un practicante de la filosofía política. No obstante, los siguientes señalamientos deben bastar para tener una idea de algunos aspectos importantes en la orientación general del proyecto constitucional-revolucionario sugerido por Ackerman.

Este proyecto visualiza un sistema legislativo "dualista" -en dos niveles. El nivel legislativo "inferior" debe corresponder a los ires y venires característicos de la "política democrática pluralista": prácticas electorales regulares, agregación de las presiones ejercidas aquí y allá por los distintos grupos que componen la sociedad, etc. En contraste, el nivel legislativo "superior", en palabras del propio Ackerman

se concibe teniendo en mente a los aspirantes a revolucionarios. Establece el recurso a procedimientos especiales con miras a determinar si una mayoría movilizada de la ciudadanía ofrece reflexivamente su apoyo a los principios que uno $u$ otro movimiento revolucionario ha pronunciado en nombre del pueblo. Aunque muchos movimientos exiguos se sienten llamados a realizar la tarea de renovación revolucionaria, los dualistas ponen énfasis en que [tan sólo] unos cuantos son escogidos por una mayoría movilizada de los ciudadanos de una nación. Por consiguiente, el sistema legislativo superior ordena un riguroso conjunto de pruebas institucionales antes de permitir que un movimiento revolucionario transforme los principios políticos fundamentales. $^{22}$

La prueba más importante a vencer es el paso del tiempo. Pero, aunque necesaria, la superación del examen del tiempo no es suficiente. Hay otras pruebas. Entre ellas, tal vez la que más destaca es la búsqueda del apoyo mayoritario a través de debates de amplio espectro en un espacio público entendido como algo más que un mecanismo para el registro y el conteo de votos. Si los "aspirantes a revolucionarios" consiguen vencer las pruebas que les impone el nivel legislativo supe-

${ }^{22}$ Bruce Ackerman, The Future of Liberal Revolution, op. cit., p. 14-5. 


\section{ERIC HERRÁN}

rior, entonces la "constitución dualista" confiere al "nuevo comienzo" una posición especial en el sistema legal. Así, a partir de ese momento y hasta la próxima revolución exitosa, "los nuevos principios han de operar como ley suprema y supervisarán [trump] los resultados de la política normal". 23

Por supuesto, algunos promotores revolucionarios verán en esta propuesta un impedimento serio para el despliegue irrestricto de su voluntad de cambio social radical. Pero entonces quizá no habrá más remedio que tratar a estos "como los criminales que son". Pues si los revolucionarios liberales deben estar dispuestos a aceptar que el entusiasmo "vanguardista" encuentre límites firmes -pero nunca inamovibles- en una estructura constitucional dualista, ello ocurre sobre la base del reconocimiento de la primacía final, en semejante estructura, del ideal que persigue el establecimiento de condiciones genuinas tanto para la deliberación como para la toma de decisión populares. ${ }^{24}$

Ya he anotado que es imposible dar cuenta aquí de la complejidad del argumento liberal-revolucionario de Ackerman, así como de sus consecuencias para, entre otros campos, la filosofía política contemporánea. De cualquier manera, ésa no ha sido nunca la intención de estas reflexiones. He querido, más bien, llamar la atención sobre el hecho-sorprendente para algunos-de que la tradición del liberalismo político es capaz de generar visiones positivas acerca del futuro del ideal revolucionario hoy en día. Lo que a mi juicio vuelve interesantes a estas visiones, es el hecho de que la exigencia revolucionaria -tal como Ackerman la entiende-no sólo aparece en ellas como ajustable al ideal de la democracia liberal, sino que la permanencia de dicha exigencia revolucionaria encuentra en las aspiraciones mismas de la democracia liberal su condición última de posibilidad.

Todo ello indica, entre otras cosas, que revolución y prudencia son dos términos que, por lo menos en sus contextos modernos, se esclarecen mutuamente. No me detendré a señalar, por bien sabida, la for-

$$
\begin{aligned}
& { }^{23} \text { Ibid., p. } 15 . \\
& { }^{24} \text { Ibid., p. } 18 .
\end{aligned}
$$


ma en que tradicionalmente se entiende que los excesos revolucionarios deben ser supervisados -y en lo posible suprimidos-en el ejercicio de la sabiduría y la sobriedad prudenciales. Congruente con mi interés en la clase de liberalismo político defendido por Ackerman, es el otro lado de esa moneda que conforman las relaciones entre la revolución y la prudencia, lo que deseo poner de relieve. Aun ahora (pero quizá ahora más que nunca), hay algo de ilegítimo en el hecho de pensar la prudencia sin preguntarse simultáneamente por la naturaleza y los alcances de esta última en el contexto de una observación cualquiera de la brecha que, en el liberalismo democrático, separa el ámbito de las promesas del campo de las realidades sociales, políticas y económicas del poder.

Ciertamente, el valor de lo que acabo de afirmar no es indiferente a la adopción de una cierta idea de la prudencia. La que tengo en mente no es muy distinta de la que habita muy cerca del centro de la filosofía política clásica. De acuerdo con esta tradición de pensamiento político, la prudencia es ciertamente moderación (en el sentido de continencia), pero no es menos saber estimar las circunstancias con miras a avanzar en la realización -parcial y nunca definitiva-de la norma. Aquí la prudencia es un tipo de cálculo inteligente, nunca idéntico a una ciencia exacta, que atiende a más de una cosa importante a la vez. En esta perspectiva, si la prudencia es moderación -en el sentido de mesura y sensatez- lo es entonces en contacto con la búsqueda permanente de algún equilibrio, siempre provisional, entre distintos fines que con frecuencia no pueden menos que aparecer ante nosotros como igualmente valiosos.

John Dunn ha recogido en forma de libro una serie de ensayos que concibió atendiendo a los vínculos entre la teoría y la responsabilidad políticas. Me parece que la cita de algunas ideas que ahí se aprecian, puede ofrecer un corolario adecuado a la argumentación que en esta oportunidad he presentado.

El mundo de la política tiene sus días apacibles e incluso sus épocas risueñas. Pero es, por su propia naturaleza, un mundo 


\section{ERIC HERRÁN}

de peligro y de rigores potenciales. No condena a los teóricos de la política a niveles inferiores de ambición moral, ya no digamos a sensibilidades morales torpes. No obstante, lo que sí hace es obligarles, so pena de extrema mala fe, a reconocer -de acuerdo con su entendimiento causal del mundo tal como es- los niveles de ambición moral que suscriben, impidiéndoles en consecuencia el sometimiento de su comprensión de la realidad del mundo a las importunidades vinculadas con la proyección de sus propios deseos. ${ }^{25}$

Por cierto la cita también habría podido servir como un epígrafe para estas reflexiones.

${ }^{25}$ John Dunn, op. cit., p. 196. 